\title{
Non-linear indices of heart rate variability during endodontic treatment
}

\author{
Milana Drumond Ramos SANTANA ${ }^{(a)}$ \\ Ivo Cavalcante PITA-NETO(a) \\ Eli Carlos MARTINIANO(b) \\ Larissa Raylane Lucas MONTEIRO(b) \\ José Lucas Souza RAMOS(b) \\ David M. GARNER(c) \\ Vitor Engácia VALENTI(d) \\ Luiz Carlos de ABREU(a)
}

(a) Faculdade de Medicina do ABC - FMABC, Laboratório de Delineamento de Estudos e Escrita Científica, Santo André, SP, Brazil.

(b)Faculdade de Juazeiro do Norte - FJN, Faculdade de Odontologia, Departamento de Saúde, Juazeiro do Norte, CE, Brazil.

(c) Oxford Brookes University, Faculty of Health and Life Sciences, Department of Biological and Medical Sciences, Oxford, United Kingdom.

(d) Universidade Estadual Paulista - UNESP, Faculdade de Filosofia e Ciências, Centro de Estudos do Sistema Nervoso Autônomo, Marília, SP, Brazil.

Declaration of Interests: The authors certify that they have no commercial or associative interest that represents a conflict of interest in connection with the manuscript.

\section{Corresponding Author:}

Milana Drumond Ramos Santana

E-mail: mildrumond@hotmail.com

DOI: 10.1590/1807-3107BOR-2016.vol30.0029

Submited: 28 Jul, 2015

Accept for publication: Oct 14, 2015

Last revision: Nov 11, 2015
Abstract: Dental treatment promotes psychosomatic change that can influence the procedure and compromise the general well-being of the patient. In this context, it highlights the importance of evaluating the function of the autonomic nervous system in individuals undergoing endodontic treatment. Thus, this manuscript aimed to analyse cardiac autonomic modulation, through non-linear indices of heart rate variability (HRV) during endodontic treatment. Analysis of 50 subjects of either sex aged between 18 and 40 years diagnosed with irreversible pulp necrosis of lower molars undergoing endodontic treatment was undertaken. We carried out fractal and symbolic analysis of HRV, which was recorded in the first session of the endodontic treatment at four intervals: T1: 0-10 min before the onset of the treatment session; T2: 0-10 min after the application of anaesthesia; T3: throughout the period of treatment; and T4: 0-30 min after the end of the treatment session. There was reduction of $\mathrm{a} 1$ in T2 compared to T1 and T4 ( $\mathrm{p}<0.0001)$. The $a 2$ index also reduced in T2 compared to T3 $(p=0.0035)$. There was an increase in the $\alpha 1 / \alpha 2$ ratio in $\mathrm{T} 4$ compared to T2 and T3 $(\mathrm{p}=0.0003)$. It was found that $0 \mathrm{~V} \%$ was significantly lower in $\mathrm{T} 2(\mathrm{p}=0.002)$, while $2 \mathrm{UV} \%$ was significantly higher $(\mathrm{p}<0.0001)$ when compared to other points in time. In conclusion, HRV is reduced during endodontic treatment, and after applying local anaesthetic the parasympathetic component of HRV increases. These data indicate that endodontic treatment acutely overcharges the heart, supporting the stress involved in this situation.

Keywords: Endodontics; Autonomic Nervous System; Cardiovascular Physiology; Heart Rate Variability.

\section{Introduction}

Dental treatment promotes psychosomatic changes which may influence the treatment plan. Some dental procedures can become extremely uncomfortable, generating anxiety and significant autonomic changes. ${ }^{1}$

Hence, it highlights the importance of evaluating the function of the autonomic nervous system (ANS) in individuals undergoing endodontic treatment, which can be achieved through heart rate variability (HRV). HRV is a simple, cheap, reliable and non-invasive tool that describes the fluctuations of the intervals between consecutive heartbeats (RR intervals). These are related to the modulation of the ANS on the sinus node. ${ }^{2}$ 
HRV can be a useful parameter for detecting even small cardiovascular changes in patients who are subjected to dental procedures, compared to other noninvasive tools. Consequently, it can assist monitoring, detect heart failure and prevent cardiovascular emergencies. ${ }^{3}$ In dental surgery, HRV has been related to malocclusion, temporomandibular disorders, dental surgery and the effects of sedatives. ${ }^{1}$

HRV can be analysed by linear and non-linear methods. Non-linear methods describe the complex fluctuations of rhythm more adequately than linear methods, as chaos theory displays some features that are consistent with those found physiologically in the human body. So, this analysis allows a more favourable assessment of the ANS condition and discrimination between a subject with normal or pathological physiology. ${ }^{4,5}$

In non-linear analysis, the approach of chaos theory considers dynamic and deterministic systems governed by non-linear methods and sensitive to the initial conditions. This form of analysis can better reflect changes in autonomic modulation of biological systems, since there is evidence that the mechanisms involved in cardiovascular regulation may interact in a non-linear manner. ${ }^{2}$

Therefore, studying non-linear behaviour, which is more representative of the physiological behaviour of individuals, can lead to a greater understanding of the influence of endodontic treatment on the ANS, and assist in the evaluation and risk stratification of these patients. As a consequence, this study was undertaken to analyse the non-linear indices of HRV in the first session of endodontic treatment.

\section{Methodology}

\section{Study population}

A total of 50 subjects from either sex aged between 18 and 40 years undergoing endodontic treatment was analysed. All volunteers were informed about the procedures and objectives of the study and, after agreeing, signed a consent letter. All study procedures were approved by the Ethics Committee in Research of the Faculty of Sciences of the Faculdade Leão Sampaio (Protocol no. 193.889) and followed National Health Resolution 466/12.

\section{Non-inclusion criteria}

Subjects were not included under the following conditions: body mass index $(\mathrm{BMI})>35 \mathrm{~kg} / \mathrm{m}^{2}$; systolic blood pressure (SBP) $>140 \mathrm{mmHg}$ or diastolic blood pressure $(\mathrm{DBP})>90 \mathrm{mmHg}$ (at rest); cardiovascular, respiratory and neurological-related disorders that prevented normal study participation; smoking; and subjects undergoing treatment with medication that influenced cardiac autonomic regulation.

\section{Initial evaluation}

Before the experimental procedure, volunteers were identified by collecting the following information: age, gender, weight, height and BMI. Weight was determined by using a digital scale (W 200/5, Welmy, São Paulo, Brazil) with a precision of 0.1 kilograma. Height was measured by using a stadiometer (ES 2020, Sanny, São Paulo, Brazil) with a precision of $0.1 \mathrm{~cm}$ and $2.20 \mathrm{~m}$ of extension. BMI was calculated by the following mathematical formula: weight/ height ${ }^{2}$, weight in kilograms and height in meters.

\section{HRV analysis}

The RR intervals were recorded using a portable heart rate monitor with a sampling rate of $1000 \mathrm{~Hz}$, then uploaded to the Polar Precision Performance program (v. 3.0, Polar Electro, Kempele, Finland). The software enabled visualisation of the heart rate and the extraction of a cardiac period (or RR interval) file downloadable in '.txt' format. Following digital filtering complemented with manual filtering for the elimination of premature ectopic beats and artifacts, at least 256 stable RR intervals were used for the data analysis. Only series with more than $95 \%$ sinus rhythm were included in the study. ${ }^{6}$ HRV was analysed at four intervals in the first session of endodontic treatment: 1) $10 \mathrm{~min}$ before the treatment, 2) $10 \mathrm{~min}$ after the administration of anaesthesia, 3) during the entire period of treatment and 4) $30 \mathrm{~min}$ after the end of the treatment.

\section{Detrended fluctuation analysis}

For analysis of the fractal properties of the heart rate, detrended fluctuation analysis (DFA) was applied to a time series of the RR intervals obtained from the participants. The procedure for the calculation of DFA consists of the following steps: 
The RR series obtained experimentally is integrated using the expression:,8

$$
\mathrm{Y}(\mathrm{k})=\sum \mathrm{i}=1 \mathrm{k}[\mathrm{RR}(\mathrm{i})-\mathrm{RR} \text { ave }]
$$

in which $\mathrm{Y}(\mathrm{k})$ is the $\mathrm{k}^{\text {th }}$ term of the integrated series $(\mathrm{k}=1,2 \ldots \mathrm{N})$; $\mathrm{RR}(\mathrm{i})$ is the $\mathrm{i}^{\text {th }}$ value of the $\mathrm{RR}$ intervals; and RRave is the mean of the RR intervals of the original series, with $\mathrm{N}$ length:

$$
\text { RRave }=1 \mathrm{~N} \sum \mathrm{i}=1 \mathrm{NRR}(\mathrm{i})
$$

The integrated time series is then divided into intervals with a length of $n,(n=1,2 \ldots N)$. In each of these intervals, the local trend of the series is calculated by a straight line of minimum squares adjusted to the data. The y-coordinate of this straight line is denominated $\mathrm{Yn}(\mathrm{k})$. The integrated series is then detrended $[\mathrm{Y}(\mathrm{k})]$, subtracting the local tendency $\mathrm{Yn}(\mathrm{k})$ in each interval. For a given interval of size $\mathrm{n}$, the size characteristic of the fluctuation for the integrated and detrended series is calculated by:

$$
F(n)=\sqrt{\frac{1}{N} \sum_{K=1}^{N}[Y(k)-Y n(k)]^{2}}
$$

This procedure is repeated for all intervals of size n, thereby obtaining a relation between the mean of the fluctuations [F(n)] and the size of the intervals (n). A linear relation on a log-log graph indicates a scale exponent law, based on the following mathematical formula:

\section{$\mathrm{F}(\mathrm{n}) \approx \mathrm{na}$}

in which $a$ is the scale exponent, which can be calculated by linear regression on a log-log grap. The following were calculated: short-term fractal exponent (a1), corresponding to a period of 4 to 11 beats; long-term fractal exponent ( $\alpha 2)$, corresponding to periods longer than 11 beats; and the a1/a2 ratio. ${ }^{9}$

When $\alpha=0.05$, there is no correlation and the signal consists of white noise; if $\alpha=1.5$, the signal resembles random walk (Brownian motion); and if $0.5<a<1.5$, there are positive correlations. If $\alpha$ is close to 1.0 it indicates a more complex (non-linear) system, and if it reaches values above 1.0 the system tends to be less complex and linear.

\section{Symbolic analysis of HRV}

Symbolic analysis was performed through distribution of the number of RR intervals into six levels (0 to 5), which transforms it into a sequence of symbols, from which there is a spatial methodology (sequence of three symbols). All possible patterns were grouped into four clusters regardless, according to the number and type of variation between subsequent symbols: 1) $0 \mathrm{~V}$ corresponds to no variation [three identical symbols, for example $(2,2,2)$ or $(4,4,4)] ; 2) 1 \mathrm{~V}$ corresponds to one variation [two consecutive symbols are equal and the remaining symbol is different, for example $(4,2,2)$ or $(4,4,3)]$; 3$) 2 \mathrm{LV}$ represents two similar variations [the three symbols form a ramp up or down, for example $(5,4,2)$ or $(1,3,4)]$; 4$) 2 \mathrm{ULV}$ represents two opposite variations [three symbols form a peak or a valley, for example $(3,5,3)$ or $(4,1,2)]$. Occurrence rates of these clusters $(0 \mathrm{~V} \%, 1 \mathrm{~V} \%, 2 \mathrm{LV} \%$ and $2 \mathrm{ULV} \%$ ) were examined. ${ }^{10}$ Previous studies involved pharmacological blockade and autonomic tests ${ }^{10}$, indicating that the index $0 \mathrm{~V} \%$ is representative of cardiac sympathetic modulation and the rate of $1 \mathrm{~V} \%$ corresponds to the simultaneous presence of sympathetic and vagal modulation. 2LV\% and $2 \mathrm{ULV} \%$ are related to cardiac vagal modulation.

\section{Endodontic treatment procedures}

After the initial evaluation, the heart monitor belt was then placed over the thorax, aligned with the distal third of the sternum, and the Polar RS800CX heart rate receiver (Polar Electro, Kempele, Finland) was placed on the wrist.

Data collection was carried out in the same room for all volunteers with the temperature between 21 and $25^{\circ} \mathrm{C}$ and relative humidity between $50 \%$ and $60 \%$; volunteers were instructed not to drink alcohol or caffeine for $24 \mathrm{~h}$ before evaluation. Data were collected on an individual basis between 08:00 and 12:00 to standardise the protocol. All procedures necessary for data collection were explained on an individual basis and the subjects were instructed to remain at rest and to avoid talking during collection.

The endodontic treatment was performed by one therapist in two sessions and followed all the steps of endodontic treatment. Firstly, anaesthesia was administered to the inferior alveolar nerve (Alphacaine 
$2 \% \mathrm{DFL}^{\circledR}$, Rio de Janeiro, Brazil). Caries removal was performed, and access to the pulp chamber obtained using spherical diamond burs (\# 1012, 1014 and 1015, KG Sorensen ${ }^{\circledR}$, Cotia, Brazil) compatible with the size of the pulp chamber, mounted on a high-rotation turbine, with air-cooled water and an Endo Z drill (Dentsply ${ }^{\circledR}$, Ballaigues, Switzerland). Isolation of the operative field was performed with a rubber dam, followed by chemo-mechanical preparation of the root canal (Dentsply ${ }^{\circledR}$ Flexofile hand file and sodium hypochlorate $2.5 \%$ ), channel drying (Paper Cones, Dentsply ${ }^{\circledR}$ ), intracanal medication (called SSWhite ${ }^{\circledR}$, Rio de Janeiro, Brazil) and placement of a cotton ball in the entrance of the temporary sealing canal (IRM Dentsply ${ }^{\circledR}$ ).

\section{Statistical analysis}

Standard statistical methods were used to calculate the means and standard deviations. The normal Gaussian distribution of the data was verified by the Shapiro-Wilk 'goodness-of-fit' test (z-value > 1.0). For parametric distributions, ANOVA was applied for a repeated measures test followed by the Bonferroni post-test. For non-parametric distributions, the Friedman test followed by the Dunns post-test was used. HRV indices were compared the between the four periods: 1) $10 \mathrm{~min}$ before the endodontic treatment, 2) 10 min after the administration of anaesthesia, 3) during the entire period of endodontic treatment, and 4) $30 \mathrm{~min}$ after the end of the treatment. Differences were considered significant when the probability of a Type I error was less than $5 \%(p<0.05)$. For statistical calculations, GraphPadStatMate v. 2.00 for Windows (GraphPad Software, San Diego, USA) was used.

\section{Results}

Table 1 shows the values for baseline diastolic (DAP) and systolic arterial pressure (SAP), heart rate (HR), mean RR interval, weight, height and BMI of the volunteers.

We noted that all patients were anxious. There was no significant statistical difference in SBP $(p=0.9584)$ or DBP $(p=0.8984)$ before and after the endodontic treatment protocol.
Table 1. Baseline diastolic (DAP) and systolic arterial pressure $(S A P)$, heart rate $(H R)$, mean RR interval, weight, height and body mass index (BMI) of the volunteers.

\begin{tabular}{lc}
\hline Variable & Value \\
\hline Age $($ years $)$ & $26.6 \pm 5.93$ \\
Height $(\mathrm{m})$ & $1.63 \pm 0.084$ \\
Weight $(\mathrm{kg})$ & $63.97 \pm 12.95$ \\
$\mathrm{BMI}\left(\mathrm{kg} / \mathrm{m}^{2}\right)$ & $23.86 \pm 3.92$ \\
HR $(\mathrm{bpm})$ & $76.95 \pm 14.39$ \\
Mean RR $(\mathrm{ms})$ & $807.08 \pm 137.32$ \\
$\mathrm{SAP}(\mathrm{mmHg})$ & $122.53 \pm 14.03$ \\
DAP $(\mathrm{mmHg})$ & $83.37 \pm 11.65$ \\
\hline Mean \pm standard-deviation. m: meters; ms: millisecond;
\end{tabular}

Mean \pm standard-deviation. $\mathrm{m}$ : meters; ms: millisecond

$\mathrm{kg}$ : kilograms; bpm: beats per minute; $\mathrm{mmHg}$ : millimeters of mercury.

Regarding fractal HRV analysis, it was observed that during the $\mathrm{T} 2$ period there was a decrease of a1 with respect to the T1 and T4 periods ( $\mathrm{p}<0.0001)$. The a 2 exponent also reduced in $\mathrm{T} 2$ compared to $\mathrm{T} 3$ $(p=0.0035)$. There was an increase in the $\alpha 1 / \mathrm{a} 2$ ratio in T4 compared to T2 and T3 $(\mathrm{p}=0.0003)$ (Table 2).

Regarding the symbolic analysis of HRV, it was observed that during $\mathrm{T} 2 \mathrm{0V} \%$ and $1 \mathrm{~V} \%$ decreased compared to other periods. $2 \mathrm{LV} \%$ reduced in $\mathrm{T} 3$ and 2UV\% increased in T2 (Table 3).

\section{Discussion}

Knowledge of the physiological responses involved in endodontic treatment is important because it provides a more appropriate treatment plan for the patient and provides greater procedural rigour for the endodontist. Analysis of fluctuations in the ANS during endodontic procedures provides information about possible risks that could arise for the cardiovascular system. The importance of investigating the effects of endodontic treatment on cardiac autonomic regulation is well understood. In this study, it was found that during endodontic treatment, HRV is reduced. Furthermore, after the administration of local anaesthetic, the parasympathetic component of HRV increased.

Despite few investigations in this area, non-linear methods help to understand system behaviour that linear models do not explain, since there is evidence that the mechanisms involved in cardiovascular regulation probably interact in a non-linear way. 
Table 2. Mean and standard deviation of fractal analysis indexes in T1, T2, T3 and T4.

\begin{tabular}{lccccc}
\hline Index & T1 & T2 & T3 & T4 & $p$-value \\
\hline DFA $\alpha 1$ & $1.01 \pm 0.26^{*}$ & $0.91 \pm 0.33$ & $0.95 \pm 0.22$ & $1.10 \pm 0.23^{*}$ & $<0.0001$ \\
DFA $\alpha 2$ & $0.90 \pm 0.14$ & $0.85 \pm 0.24$ & $0.92 \pm 0.10^{*}$ & $0.91 \pm 0.10$ & 0.0035 \\
$\alpha 1 / \alpha 2$ & $1.13 \pm 0.34$ & $1.03 \pm 0.36^{* *}$ & $1.02 \pm 0.31^{* *}$ & $1.25 \pm 0.37$ & 0.0003 \\
\hline${ }^{*} p<0,05:$ Vs. T2. & & & & & \\
$*_{p}^{*}<0,05:$ Vs.T4. & & & & &
\end{tabular}

Table 3. Mean and standard deviation of symbolic analysis indexes in T1, T2, T3 and T4.

\begin{tabular}{lccccc}
\hline Index & T1 & T2 & T3 & T4 & -value \\
\hline OV\% & $17.78 \pm 13.82^{* *}$ & $12.53 \pm 9.52$ & $20.14 \pm 14.28^{* *}$ & $18.47 \pm 9.98^{* *}$ & 0.002 \\
1V\% & $44.08 \pm 7.02^{* *}$ & $41.32 \pm 7.80$ & $44.93 \pm 6.03^{* *}$ & $45.77 \pm 4.58^{* *}$ & 0.0013 \\
2LV\% & $15.81 \pm 7.39^{*}$ & $15.71 \pm 5.51^{*}$ & $10.79 \pm 5.67$ & $14.73 \pm 5.51^{*}$ & $<0.0001$ \\
2UV\% & $22.29 \pm 11.18^{* *}$ & $30.41 \pm 13.35$ & $24.11 \pm 0.95^{* *}$ & $21.01 \pm 10.10^{* *}$ & $<0.0001$ \\
\hline
\end{tabular}

${ }^{*} p<0,05: V_{s}$. T3.

${ }^{* *} \mathrm{p}<0,05:$ Vs.T2.

Chaos theory describes behaviour of elements that are extremely sensitive to initial conditions, are difficult to replicate, but are deterministic. ${ }^{2}$ In this sense, the study of HRV through non-linear methods has received increasing interest, and fractal and symbolic analysis are able to explore this relationship. ${ }^{11}$

According to Tulppo et al., ${ }^{12}$ fractal exponents seem to be able to detect subtle changes in the dynamics of RR intervals more favourably than those obtained with conventional spectral analysis. Sousa et al. ${ }^{13}$ also concluded that fractal analysis of HRV is more sensitive than the analysis of time and frequency domain indices to assess the effects of postural change manoeuvres on cardiac autonomic modulation.

In the analysis of fractals, exponent a1 ranges between 0.5 and 1.5. If $a$ is close to 1.0, it indicates the predominance of a more complex non-linear system; if it is above 1, the system tends to be less complex and tends to a linear system, indicating a poor prognosis, or physiological pathology. ${ }^{9}$ The reduction of the fractal index indicates insufficient adaptation of the ANS and involves the presence of a physiological malfunction in the individual. Studies in patients with chronic obstructive pulmonary disease (COPD) found a reduction in the a1 fractal exponent of this population. In this sense, the index can be applied for risk stratification and assessment of manifestations of systemic diseases. ${ }^{7}$
There was a decrease in the value of a1 in T2 compared to T1 and T4, indicating an increase of vagal modulation after applying the local anaesthetic, given that the values tended to linearity.

Furthermore, the values of the $\mathrm{a} 1$ and $\mathrm{a} 2$ exponents are close to unity and a1 was higher than $a 2$, which is also a characteristic of healthy individuals, as found in the studies of Lombardi ${ }^{14}$ and Acharya. ${ }^{15}$

Symbolic analysis proposes conversion of large time series of a sequence of samples, groups them into 'symbols' and studies the dynamics of the symbols instead of the original samples. Symbolic analysis applied to HRV is able to distinguish different physiological conditions in individuals. ${ }^{16}$

It was found that $0 \mathrm{~V} \%$ was significantly lower in $\mathrm{T} 2$, while $2 \mathrm{UV} \%$ was significantly higher when compared to other time points. There was an increased parasympathetic modulation after application of the local anaesthetic, identified by the predominance of standard 2UV\%. Guzzetti et al. ${ }^{17}$ assessed HRV in three combinations of anaesthetics during neurosurgical procedures through linear and non-linear indices. Patients were randomly divided into three groups according to type of anaesthesia: fentanyl with sevoflurane, sevoflurane with remifentanil and propofol with remifentanil. It was observed that all anaesthetic combinations were characterised by a reduced HRV. The authors concluded that symbolic 
analysis seems to be able to highlight the differences of both sympathetic (slow) and vagal modulation (fast) amongst anaesthetics.

There was a decrease in $2 \mathrm{LV} \%$ in $\mathrm{T} 3$ when compared to the other periods. This indicates that during endodontic treatment, $\mathrm{HRV}$ is reduced. These results are in agreement with those found by Santana et al. ${ }^{18}$ that evaluated the acute effects of endodontic treatment on cardiac autonomic modulation through linear indices (time and frequency) of HRV.

The reduction of HRV after local anaesthetic in dental surgery was also observed in the studies of Matsumura et al. ${ }^{19}$ and Miura et al. ${ }^{20}$ These authors observed that the LF/HF ratio decreased during local anaesthesia. However, when comparing HRV before and after surgery in patients who received local anaesthesia with or without intravenous sedation in dental implant surgery, Morino et al..$^{21}$ observed that HF, LF and LF/HF ratio did not differ between study times (pre- and post-operative).

Svorc et al. ${ }^{22}$ corroborated this study: the authors concluded that anaesthesia increased HRV, with increased parasympathetic modulation of the heart determining the effect of ketamine/xylazine with pentobarbital.

HRV has been a useful parameter for detecting even minor cardiovascular changes over other non-invasive tools for patients who are subjected to endodontic procedures, since there was no variation before and after the sessions in the systolic and diastolic blood pressures. This result confirms the findings of Montebugnoli et al., ${ }^{3}$ where there was no difference in systolic and diastolic blood pressure in the four study periods (early in the dental surgery, immediately after anaesthesia, during dental extraction and $5 \mathrm{~min}$ after tooth extraction). However, $\mathrm{HR}$ values were significantly different in three of the four periods, and HRV values were significantly different for all four test periods.

Previous studies have reported an increase in blood pressure and HR associated with infiltrative anaesthesia and blocking the inferior alveolar nerve with lidocaine and epinephrine, but no clinically relevant effect on cardiovascular parameters. ${ }^{23,24,25}$

Anxiety and fear of endodontic treatment were reported by a large number of patients during this study. These emotions can generate significant autonomic changes and determine physiological changes related to sympathetic nervous system activity, expressed as pallor, sweating palms, trembling, communication difficulties and tachycardia. ${ }^{26}$ Nevertheless, this study found that the effect of the local anaesthetic caused suppression of the cardiac sympathetic nervous system.

With regards to the effects of stress, anxiety and depression in the modulation of ANS, Langewitz et al. ${ }^{26}$ evaluated the effect of mental stress on the ANS through $\mathrm{HRV}$, where the decrease of some variables of HRV was observed. According to Morino et al., ${ }^{21}$ analysis of HRV is useful in assessing stress and anxiety.

There are potential pitfalls in this study. The age range was wide (18 to 40 years old). However, exclusion criteria (smoking; individuals with cardiorespiratory disease, neurological disorders and other related impairments that prevented the subject performing the procedures; and treatment with drugs that influence cardiac autonomic regulation) sought to ensure normative standards in relation to the sample selection. The first session of endodontic treatment was analysed in order to prevent conditioning of the patient, thus it was standardised for patients who had already sustained previous endodontic treatment.

Few studies describe autonomic modulation during endodontic treatment, and this study is the first to analyse the non-linear indices of HRV in endodontics. The oscillations in RR intervals analysed through HRV provide important variables to stratify cardiovascular risk in endodontic treatment. Consequently, further studies are suggested to investigate HRV as a useful tool for obtaining parameters to stratify cardiovascular risk in the various dental specialties.

\section{Conclusion}

During local anaesthesia for endodontic treatment, the parasympathetic component of cardiac modulation increases compared to before surgery and during surgical procedures.

\section{Acknowledgements}

This study received financial support from Faculdade de Medicina do ABC - FMABC and Faculdade de Juazeiro do Norte - FJN. 


\section{References}

1. Santana MDR, Sousa ACA, Abreu LC, Valenti, VE. Association between oral variables and heart rate variability. Int Arch Med. 2013 Dec 27;6(1):49. doi:10.1186/1755-7682-6-49

2. Vanderlei LCM, Pastre CM, Hoshi RA, Carvalho TD, Godoy MF. Noções básicas de variabilidade da frequência cardíaca e sua aplicabilidade clínica. Rev Bras Cir Cardiovasc. 2009;24(2): 205-17. doi:10.1590/S0102-76382009000200018

3. Montebugnoli L, Servidio D, Miaton RA, Prati C. Heart rate variability: a sensitive parameter for detecting abnormal cardiocirculatory changes during a stressful dental procedure. J Am Dent Assoc. 2004;135(12):1718-23. doi:10.14219/jada.archive.2004.0125

4. Task Force of the European Society of Cardiology and the North American Society of Pacing and Electrophysiology. Heart rate variability: standards of measurement, physiological interpretation and clinical use. Circulation. 1996;93(5):1043-65. doi:10.1161/01.CIR.93.5.1043

5. Valenti VE. The recent use of heart rate variability for research. J Human Growth Dev. 2015;25(2):137-9. doi:10.7322/jhgd.102991

6. Abreu LC. Heart rate variability as a functional marker of development. J Human Growth Dev. 2012;22(3):279-82. doi:10.7322/jhgd.46712

7. Carvalho TD, Pastre CM, Godoy MF, Fereira C, Pitta FO, de Abreu LC, et al. Fractal correlation property of heart rate variability in chronic obstructive pulmonary disease. Int J Chron Obstruct Pulmon Dis. 2011 Jan 6;6:23-8. doi:10.2147/COPD.S15099

8. Tulppo MP, Kiviniemi AM, Hautala AJ, Kallio M, Seppänen T, Mäkikallio $\mathrm{TH}$, et al.Physiological background of the loss of fractal heart rate dynamics. Circulation. 2005 Jul 19;112(3):314-9. doi:10.1161/CIRCULATIONAHA.104.523712

9. Makikallio TH, Høiber S, Køber L, Torp-Pedersen C, Peng CK, Goldberger AL, et al. Fractal analysis of heart rate dynamics as a predictor of mortality in patients with depressed left ventricular function after acute myocardial infarction. Am J Cardiol. 1999;83(6):836-9. doi:10.1016/S0002-9149(98)01076-5

10. Porta A, GuzzettiS, Montano N, Furlan R, Pagani M, Malliani A, et al. Entropy, entropy rate, and pattern classification as tools to typify complexity in short heart period variability series. IEEE Trans Biomed Eng. 2001;48(11):1282-91. doi:10.1109/10.959324

11. Correa PR, Catai AM, Takakura IT, Machado MN, Godoy MF. Heart rate variability and pulmonary infections after myocardial revascularization. Arq Bras Cardiol. 2010;95(4):448-56. doi:10.1590/S0066-782X2010005000123

12. Tulppo MP, Mäkikallio TH, Seppänen T, Shoemaker K, Tutungi E, Hughson RL, et al. Effects of pharmacological adrenergic and vagal modulation on fractal heart rate dynamics. Clin Physiol. 2001;25(5):515-23. doi:10.1046/j.1365-2281.2001.00344.x

13. Souza ACA, Cisternas JR, Abreu LC, Roque AL, Monteiro CBM, Adami F, et al. Fractal correlation property of heart rate variability in response to the postural change maneuver in healthy women. Int Arch Med. 2014 May 15;7:25. doi:10.1186/1755-7682-7-25. eCollection 2014.

14. Lombardi F. Chaos theory, heart rate variability and arrhythmic mortality. Circulation. 2000 Jan; 101(1): 8-10. doi:10.1161/01.CIR.101.1.8

15. Acharya UR, Joseph KP, Kannathal N, Lim CM, Suri JS. Heart rate variability: a review. Med Biol Eng Comput. 2006;44(12);1031-51. doi:10.1007/s11517-006-0119-0

16. Porta A, Gnecchi-Ruscone T, Tobaldini E, Guzzetti S, Furlan $\mathrm{R}$, Malliani A, et al. Symbolic analysis of short-term heart period variability during graded head-up tilt. Comput Cardiol. 2006;33:109-12. doi:10.1007/s11517-006-0119-0

17. Guzzetti S, Bassani T, Latini R, Masson S, Barlera S, Citerio $\mathrm{G}$, et al. Autonomic cardiovascular modulation with three different anesthetic strategies during neurosurgical procedures. Minerva Anestesiol. 2015;81(1):3-11.

18. Santana MDR, Sousa ACA, Assis EV, Abreu LC, Valenti VE. Changes in Heart Period During Endodontic Treatment. Exp Clin Cardiol. 2014;20(8):3389-98.

19. Matsumura K, Miura K, Takata Y, Kurokava H, Kajiyama $\mathrm{M}$, Abe I, et al. Changes in blood pressure and heart rate variability during dental surgery. Am J Hypertens. 1998;11(11):1376-80. doi:10.1016/S0895-7061(98)00157-5

20. Miura K, Matsumura K, Nakamura Y, Kurokava H, Kajiyama M, Takata y. Suppression of cardiac sympathetic nervous system during dental surgery in hypertensive patients. Hypertens Res. 2000;23(3):207-12. doi:10.1291/hypres.23.207

21. Morino M, Masaki C, Seo Y, Mukai C, Mukaibo T, Kondo $\mathrm{Y}$, et al. Non-randomized controlled prospective study on perioperative levels of stress and dysautonomia during dental implant surgery. J Prosthodont Res. 2014;58(3):177-83. doi:10.1016/j.jpor.2014.03.006

22. Svorc P Jr, Bacová I, Svorc P, Buzga M. Autonomic nervous system under ketamine/xylazine and pentobarbital anaesthesia in a Wistar rat model: a chronobiological view. Prague Med Rep. 2013;114(2):72-80. doi:10.14712/23362936.2014.25

23. Ketabi M, Shamami MS, Alaie M, Shamami MS. Influence of local anesthetics with or without epinephrine $1 / 80000$ on blood pressure and heart rate: a randomized double-blind experimental clinical trial. Dent Res J (Isfahan). 2012;9(4):437-40.

24. Faraco FN, Kawakami PY, Mestnik MJ, Ferrari DS, Shibli JA. Effect of anesthetics containing lidocaine and epinephrine on cardiovascular changes during dental 
implant surgery. J Oral Implantol. 2007;33(2):84-8. doi:10.1563/0.845.1

25. Meral G, Tasar F, Sayin F, Saysel M, Kir S, Karabulut E. Effects of lidocaine with and without epinephrine on plasma epinephrine and lidocaine concentrations and hemodynamic values during third molar surgery. Oral Surg
Oral Med Oral Pathol Oral Radiol Endod. 2005;100(2)e25-30. doi:10.1016/j.tripleo.2005.03.031

26. Langewitz, W, Ruddel, H, Schachenger H. Reduced parasympathetic cardiac control in patients with hypertension at rest and under mental stress. Am Heart J. 1994; 127(1):122-8. doi:10.1016/0002-8703(94)90517-7 